\title{
Cloud Based Software Defined Wireless Body Area Networks Architecture for Virtual Hospital
}

\author{
Md Al Shayokh \\ Department of IT Convergence \\ Engineering \\ Kumoh National Institute of \\ Technology \\ Gumi, South Korea \\ shayokh@kumoh.ac.kr
}

\author{
Jin Woo Kim \\ Department of IT Convergence \\ Engineering \\ Kumoh National Institute of \\ Technology \\ Gumi, South Korea \\ rerua@kumoh.ac.kr
}

\author{
Soo Young Shin \\ Department of IT Convergence \\ Engineering \\ Kumoh National Institute of \\ Technology \\ Gumi, South Korea \\ wdragon@kumoh.ac.kr
}

\begin{abstract}
Recent developments in wireless communication inflate a new area of research named as Wireless Body Area Networks. WBANs enhance the concept of virtual hospital with the help of cloud computing (CC) technology. Still numerous technical challenges could be found to integrate Cloud Computing (CC) and WBANs. From networking perspective, Software Defined Networking (SDN) becomes popular for its centralized nature to manage complex network infrastructure. In this paper, the concept of cloud enabled virtual hospital architecture based on software defined networking has been proposed and studied for wireless body area networks. This paper highlights a new, simplified architecture for virtual hospital concepts harnessing the benefits of software defined networking to mitigate the complexity of cloud architecture and its integration with WBAN.
\end{abstract}

\section{Categories and Subject Descriptors}

B.7.1 [Types and Design]: Advanced Technologies; C.2.3 [Network Operations]: Network Management

\section{General Terms}

Management, Design, Security, Theory.

\section{Keywords}

WBAN, Cloud Computing, SDN, Virtual Hospital, e-health monitoring

\section{INTRODUCTION}

Advancements in the field of wireless and mobile communication technologies especially in low powered communication devices make the concept of virtual hospital or e-healthcare and management system very popular. Data management for the collected data from numerous WBAN devices in large scale still remains as a matter of concern and research. Having standards such as low power, limited memory, energy and computational time, the requirement of powerful and scalable high performance computing immense storage infrastructure for data processing and storage become a vital point in terms of medical data analysis. With the growth of internet in today's world the concept of virtual hospital become very popular [1]. This concept is basically an integration of multi discipline such as network technologies, information technologies, clinical medicines etc. Network technologies play a significant role in virtual hospital system. Cloud computing (CC) is one of the emerging technologies which provide tremendous computing flexibility, storage and software services in a virtual platform. Cloud can manage large scale server cluster, storage server and other virtualized resources at low cost. Cloud computing could be described as the virtualized computational resource pool which could calculate and expand to more computation resources [2]. As technologies expands through time, the networking infrastructure becoming complex and hard to manage. For that, new idea came up to pull out computational and memory intensive network management operation which also gives hope to change the limitations of current network infrastructure. This concept is widely known as Software Defined Networking (SDN). SDN divides vertical integration by separating control plane from the routers which forwards the data plane. By separating control and data plane, networking devices' design become simple and using control logic with the help of a centralized controller makes network reconfiguration easier than before. Data and computational modules storage facility as well as processing facility in cloud make cloud computing devices reasonably cheap [3]. It could be predicted that the integration of cloud computing and WBANs is going to facilitate a new cost effective and data driven system which would be able to boost up the concept of virtual hospital for the upcoming days. Integration of these technologies will include advantages such as higher functionalities and services, increased efficiency, higher reliability etc. Although cloud based WBAN platforms are still in its outset stage, there would be several technical issues and challenges to intensify this platform into the research area [4]. In this paper, an architecture for the virtual hospital has been proposed with the integration of software defined networking in the cloud computing and WBANs to augment the storage and computational capability of cloud computing with the simplicity and flexibility of SDN which is crucial to implement the pervasive hospital system. The remainder of this paper is organized as follows. Section 2 will discuss about the integration of cloud computing with WBANs having SDN capabilities. Section 3 provides an architectural overview of the proposed architecture with functionalities. Finally, section 4 will present possible research directions with concluding remarks. 


\section{INTREGATION OF SOFTWARE DEFINED CLOUD COMPUTING WITH WBANs}

WBANs have gained popularity as an imperative technology for remote healthcare system as well as for the virtual hospitalization sector also. Miniaturized wearable devices used for WBANs are able to collect vital signals and movements and could forward them to dedicated medical servers. As cloud computing has advantage in terms of scalability, elasticity and cost efficiency, it could be a better choice to use these advantages with the integration of WBANs [5]. Moreover, SDN which renowned as an emerging architecture could add another dimension with cloud computing in terms of adaptability, high bandwidth capability as well as manageability. As different mobile applications in healthcare system getting popular day by day and medical video streaming become prime concern, cloud computing enabled SDN could be a part of solution to this concern in terms of QoS and other functionalities. The necessity of cloud computing having SDN capabilities in WBANs could be benefited as following aspects:

* Data Handling: Continuous data generated by the WBANs sensors have to be collected, stored and processed efficiently. Data management system has to be distributed in terms of space and time. Cloud computing could make possible this management system by enabling a seamless access to a massive scale storage.

- Data Analysis: Collected data could be imported for analyzing and modeling as well as making medical decision based on medical decision management (MDM) system.

- Centrally Manageable System: By using SDN in networking, network intelligence is going to be centralized with the help of SDN controllers which maintain a global view of network. Moreover, QoS mechanism could be programmed by using OpenFlow.

* Cloud Computing Applications: Developing massive scale WBAN application seems to be a complex task which includes large scale programming capability where coordination of collecting, storing and processing of distributed wearable body sensors data are prime concern. Due to these issues, software as a service (SaaS) approaches has proposed for further exploiting with the integration of WBAN frameworks.

Here, a SDN based conceptual architecture has been demonstrated with the integration of CC and WBANs in figure 1. In this figure, mobile devices will act as a gateway for the WBANs and could access the unified SDN network via Wi-Fi or cellular networks for coordinating with cloud. Later, requested tasks would be performed in cloud with the help of virtual machine (VM) as per the request of the patients or stake holders.

\section{PROPOSED ARCHITECTURE}

The prime goal of this paper is to design software based cloud enabled architecture for WBANs in different environments where the virtual hospital concept concerned. In this part, the proposed framework for the virtual hospital based on software defined networking for cloud computing will be discussed briefly with system functionalities.

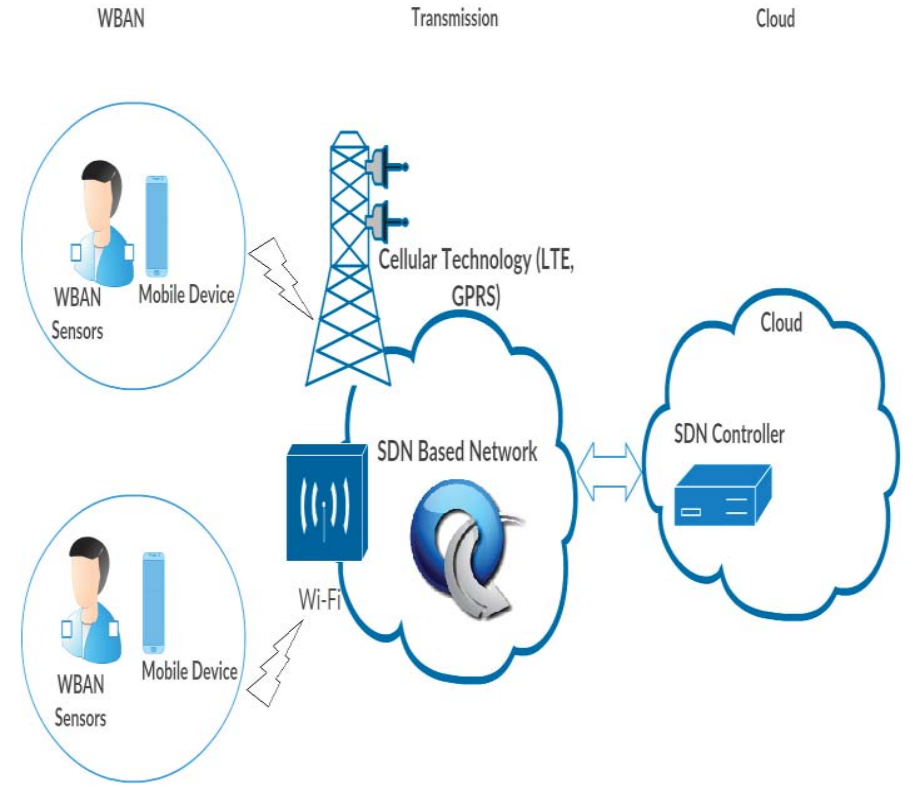

Figure 1: Conceptual architecture for WBAN with SDN based Cloud

\subsection{SDN Based Cloud Integrated WBAN Architecture}

Figure 2 illustrates a general architectural framework for virtual hospital in which SDN based cloud computing has been integrated in WBANs. The system can be viewed as the collaboration of WBANs, virtualized cellular/wireless networks, cloud services and Users. WBANs are deployed on human body to collect details of quantitative real-time body signs such as heart rate, blood pressure, temperature, etc. extending the health care beyond the confines of limited geographical area. With the existence of WBANs, a huge amount of medical data is sent to be stored in cloud knowledge base via mobile device used as a gateway. The cloud infrastructures have the necessary resources such CPU power, memory space and bandwidth to provide services including medical video streaming, alarming system, GPS and GIS services and decision support system from the knowledge base. The knowledge base stores the raw data captured from wearable body area network devices and exchanges its data with data mining module for knowledge discovery. The large data generated and collected from body area networks can be analyzed by exploiting data mining techniques to uncover meaningful and important knowledge for decision making. The discovered knowledge daily increases to help in fostering hospital operations and enhance quality of services for patients, as well as helps researchers for medical innovation. For instance, urgent health concerns can be handled based on the information extracted from knowledge base. A number of techniques and methods such as classification, cluster analysis, sequential data mining, association rule mining, clinical pathways analysis and other sophisticated approaches can be used to mine valuable knowledge from medical data for decision-making activities. The integration of WBAN technology enabled by cloud computing can also increase the quality of service for virtual hospitals, particularly in reducing errors by learning from previous error patterns and responding fast for urgent cases. Different users such as hospital staff, families and government can access the cloud data on web and from mobile entities. It has been found after a rigorous and 
extensive research that few research works such as [7-9] have been found in literature to investigate the integration of WBAN and cloud for an

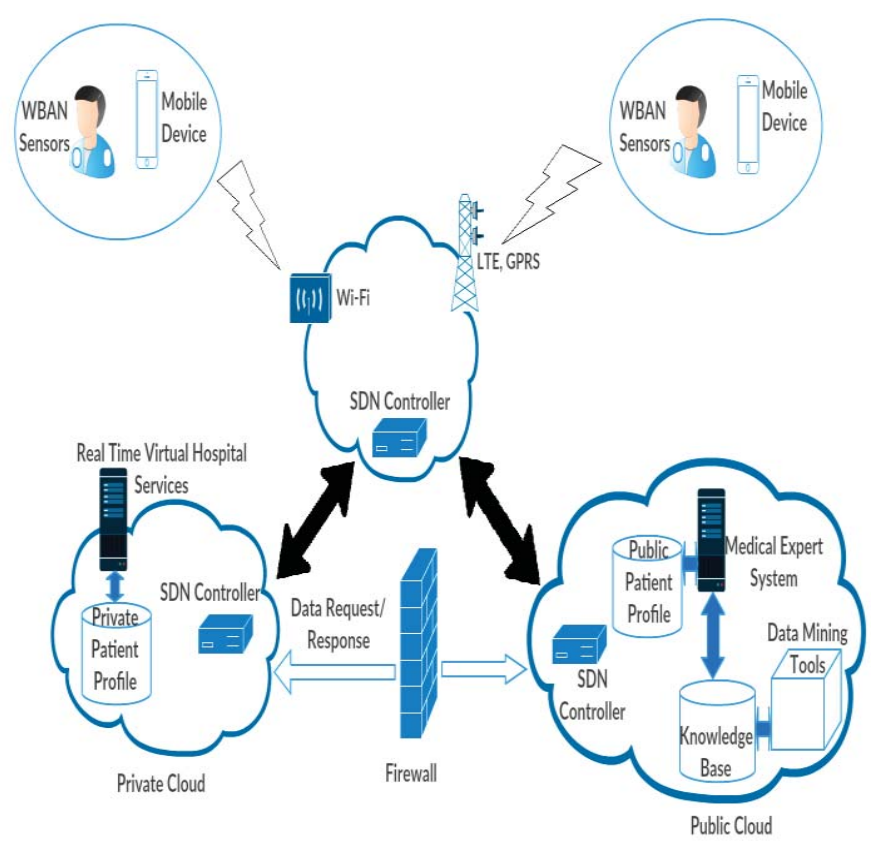

Figure 2: Framework for SDN enabled Cloud based Virtual Hospital Systems

optimized, pervasive health care systems but none of them focused open, programmable, flexible, QoS-aware system that can guarantee quality of service requirements which is necessary for health information. In this paper, prime aspects and vital issues has been suggested for virtual hospital services and applications to exploit SDN capabilities..

\subsection{Standards and Technologies as an Enabler for large-scale Virtual hospital}

For the proposed architecture, appropriate WBANs standards, benefits of cloud computing integrated with WBANs, Data Mining and the innovation that SDN leverages with cloud services has been discussed and pinpointed.

* WBAN standards: low power consumption remains a critical issue in WBANs which has its root in communication standards and protocols. Nowadays, many protocols and standards has been introduced for WBANs such as IEEE 802.15.1, IEEE 802.15.4, IEEE 802.15.6, Insteon, Z-Wave, ANT, RuBee and radio frequency identification (RFID). It has been found that IEEE 802.15.4 standard is suitable for WBANs due to low-power consumption, fast and scalable capability [6].

* Cloud: A combination of 2 or more cloud forms hybrid clouds where the entities remain unique. A hybrid cloud deployment will share technology but the information, applications, etc. will be kept apart. Healthcare providers deploy a hybrid cloud for disaster recovery of diagnostic images or clinical systems. Important medical data and applications such as GIS deployment can be deployed on a local private cloud to guarantee security while operations related to system development, upgrade, and testing can be carried out on a public cloud. Increased levels of control over the data are more apparent in a hybrid model, as applications keep a degree of separation between facilities while utilizing economies of scale in the infrastructure.

* Data mining: From figure 2, the data mining module in the cloud performs data mining techniques and algorithms to extract meaningful data stored in cloud, which is utilized in medical guidelines, resource management, and decision making. The system helps in automatic data gathering from body via WBANs devices to make data available in cloud for users and various stake holders and help in managing hospital resources and increase the quality of health care services [6]. The health employees can predict the symptoms and effects of certain health problems of the patient based on the knowledge contained in the knowledge base. $S D N$ : SDN accelerates innovation by breaking the bond between proprietary hardware and control/application software. Coupled with Network Function Virtualization (NFV), SDN seek to leverage automation and virtualization to achieve greater agility while reducing both OPEX (Operational Expenditure) and CAPEX (Capital Expenditure) [7]. Whereas NFV is intended to optimize the deployment of network functions, OpenFlow-based SDN is focused on optimizing the underlying networks. In our system, various cellular and wireless networks such as LTE, 3G, Wi-Max, Wi-Fi and GPRS share the same underlying physical infrastructure by network function virtualization (NFV) under SDN/OpenFlow. The SDN/OpenFlow provides with a system that is programmable, flexible and multi-protocol, hiding the heterogeneity (LTE, 3G, Wi-Max, Wi-Fi and GPRS), complexity and flow behaviors.

\subsection{QoS Support and Functionalities in SDN based Cloud Enabled WBANs}

From figure 2, the medical situation of patient could be followed up on a regular basis by WBAN, virtualized cellular/wireless networks and successfully stored in cloud based on the location of patient and urgency of the case. The patient can directly communicate with caregivers or relatives over video conference. The profile and medical data of patient is stored on public or private cloud according to their sensitivity. Different kinds of users like families and doctors can view patient's data using web or mobile devices according to service priority or alarming system. With cloud support, the mobile devices of medical staff will easily exhibit richer mobile video streaming from remote cameras. The cloud services also provide GIS deployment for patient location tracking. The flowchart of a virtual hospital system with SDN based mobile cloud capability is given in Fig. 3. QoS supporting functionalities has been focused from WBAN to users via cloud for patients in different locations and using different virtual services. As figure 3 flow chart shows, the cluster head of body sensors collect data and send to mobile gateway, which classify the flows according to the QoS classes. Based on the mobile QoS classification, the network allocates the bandwidth differently to handle each classified flow. For 
simplicity, we defined the category as urgent and non-urgent flows. Urgent flows are flows related to abnormal values of heart beat, blood pressure and etc measures reported by sensors while normal readings of sensors are categorized under non-urgent flows. Each body sensor is predefined with ID, type, normal and abnormal reading ranges. If the abnormal reading is encountered while mobile device checks the incoming date from sensors against its defined threshold, it invokes QoS mechanism. Assume that $\mathrm{B}$ is the total bandwidth, and $\mathrm{C}$ is the sub-bandwidth of the total. Urgent packets use the exclusively allocated bandwidth B-C, but shares $\mathrm{C}$ with non-urgent packets. For the indoor patients such as at home and hospital, various wireless technologies can help us for locating the real and exact location. For instance, time difference of arrival (TDOA) and time of arrival (TOA) and other location based technology could be implemented for further determine patient activity by data fusion technology. For outdoor patients, mobile device could be used to collect and submit the patient's data to the cloud. The alert messaging system immediately informs various medical stakeholders and families in the time of emergency according to GPS or GIS location information.

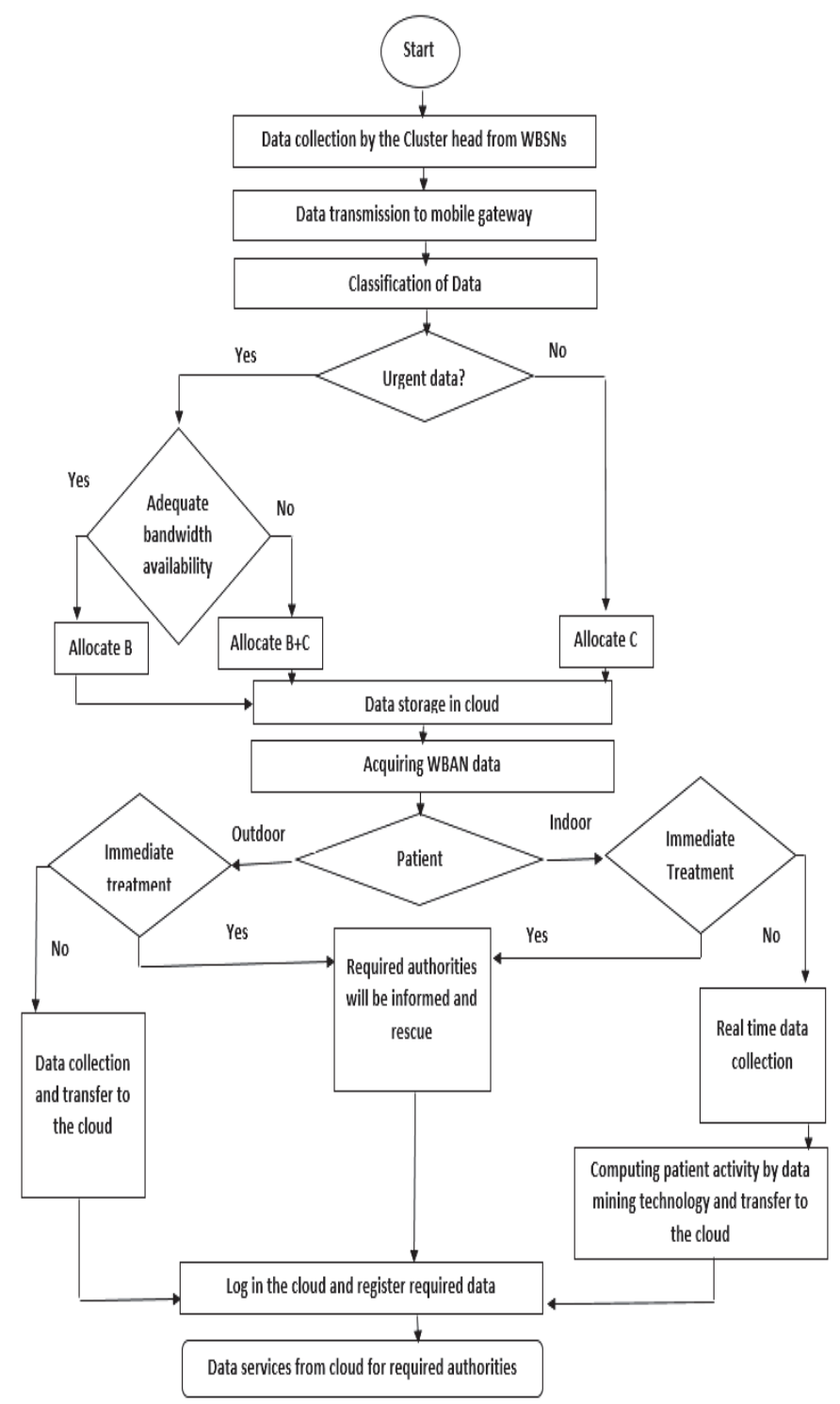

Figure 3: Logical flowchart for proposed virtual hospital with the integration of SDN based CC

\section{OPEN RESEARCH ISSUES IN WBAN AND CONCLUSION}

Integration of BAN with Cloud Computing emerges as recent research area where various research issues do still exist. Cloud resource allocation mechanisms as well as cloud security remains an issue for research where adaptation of WBAN with CC could be a great challenge in terms of methodologies and tools development. In this paper, generalized software defined cloud based architecture for virtual hospital has been proposed. Specifically, functionality, reliability and uniqueness of knowledge based data mining technology has been provided in this paper. Finally, future possible open research issues and challenges have been discussed to provide an interesting and enormous research effort for near future.

\section{ACKNOWLEDGEMENTS}

This research was supported by the MSIP (Ministry of Science, ICT and Future Planning), Korea under the CITRC (Convergence Information Technology Research Center) support program (IITP2015-H8601-15-1011).

\section{REFERENCES}

[1] Ghada Almashaqbeh, Thaier Hayajneh, Athanasios, Bassam J. Mohd. "QoS Aware Health Monitoring System using Cloud based WBANs". Journal of Medical Systems, 2014. DOI=http://dl.acm.org/citation.cfm?id=2685241

[2] C. Doukas, T. Pliakas, I. Maglogiannis. "Mobile Healthcare information Management Utilizing Cloud Computing and Android OS”. Proceedings of IEEE Eng. Med. Bio. Soc., 1037-1040, 2010

[3] Bruno Astuto A. Nunes, Marc Mendonca, Xuan-Nam Nguyen, Katia Obraczka, Thierry Turletti. "A Survey of Software Defined Networking: Past, Present and Fututre programmable Networks". IEEE Communications Surveys \& Tutorials, Vol. 16, No.3, 1617-1634, 2014.

DOI=http://ieeexplore.ieee.org/stamp/stamp.jsp?tp=\&arnum ber $=6739370$

[4] Gian Carlo Fortino, Giuseppe Di Fatta, Mukaddim Pathan, Athanasios V. Vasilakos. "Cloud Assisted Body Area Networks: State of the Art and Future Challenges". Wireless Networks, 1925-1938, 2014.

DOI=http://link.springer.com/article/10.1007\%2Fs11276014-0714-1\#page-1

[5] W. Yi, M.B Blake. "Service Oriented Computing and Cloud Computing: Challenges and Opportunities". IEEE Internet Computing, Vol. 14, No.6, 72-75, 2010.

DOI=http://ieeexplore.ieee.org/stamp/stamp.jsp?tp=\&arnum ber $=5617062$

[6] Gian Carlo Fortino, Raffaele Gravina, Antonio Guerrieri, Giuseppe Di Fatta. "Engineering Large Scale Body Area Network Applications". Proceedings of Bodynet Conference (Boston, United States, September 30-October 2, 2013) DOI=http://dl.acm.org/citation.cfm?id=2555402

[7] G Fortino, M Pathan, G Di Fatta. "BodyCloud: Integration of Cloud Computing and body sensor networks", IEEE International Conference on Cloud Computing Technology and Science (CloudCom), Dec.2012 DOI=http://dx.doi.org/10.1109/CloudCom.2012.6427537 